\title{
CONTROLE ROBUSTO POR REALIMENTAÇÃO LINEARIZANTE PARCIAL DE BIOREATORES EM MODO DE OPERAÇÃO DESCONTÍNUA COM ALIMENTAÇÃO
}

\author{
Guilherme Pimentel ${ }^{*}$ \\ guilherme.araujopimentel@umons.ac.be
}

\author{
Daniel Coutinho $^{\dagger}$ \\ daniel.coutinhoeufsc.br
}

\author{
*Service d'Automatique - UMONS \\ Boulevard Dolez 31, 7000 Mons, Belgique \\ ${ }^{\dagger}$ Departamento de Automação e Sistemas - UFSC \\ Florianópolis-SC, Cx. Postal 476, CEP 88040-900, Brasil.
}

\begin{abstract}
Robust Partial Feedback Linearizing Control of Bioreactors in Fed-Batch Mode

A methodology for the robust control design of a class of bioreactors operating in fed-batch mode is proposed. Firstly, a general model is presented to describe the growing dynamics of the bacteria Escherichia Coli and the yeast Saccharomyces Cerevisiae cultures, which are the most employed microorganisms applied in biotechnological industry. From this model, the paper proposes a control law that regulates the by-product concentration in small levels (i.e., close to zero) aiming to maximize the biomass concentration. To this end, the nonlinear dynamics of the bioreactor is rewritten in terms of time-varying parameters (quasi-LPV approach) in order to apply the linear matrix inequality framework for designing the free dynamics resulting from a partial feedback linearizing strategy. The proposed design conditions provide the robust stability of the closed-loop system while ensuring some performance w.r.t. disturbance signals. To verify the behavior of the closed-loop system, several simulation based tests are carried out to evaluate the controller performance against some recent strategies proposed in the specialized literature.
\end{abstract}

Artigo submetido em 30/11/2010 (Id.: 1225)

Revisado em 25/03/2011, 18/06/2011

Aceito sob recomendação do Editor Associado Prof. Luis Fernando Alves Pereira
KEYWORDS: bioreactor, fed-batch cultures, feedback linearization, robust control, LMIs.

\section{RESUMO}

Uma metodologia para o projeto de controladores robustos foi desenvolvida para bioreatores operando em modo descontínuo com alimentação. Inicialmente, apresenta-se um modelo geral que descreve a dinâmica do crescimento da bactéria Escherichia Coli e da levedura Saccharomyces Cerevisiae que são atualmente os dois microorganismos mais utilizados na indústria biotecnológica. A partir do modelo dinâmico da cultura de microorganismos, busca-se projetar uma lei de controle que mantenha a concentração do produto secundário em níveis próximos a zero visando, desta forma, maximizar a produção de biomassa. Com este objetivo, a dinâmica não linear é descrita em termos de parâmetros variantes no tempo (abordagem quasi-LPV) possibilitando a utilização da formulação por desigualdades matriciais lineares para o projeto da dinâmica livre resultante da aplicação de uma lei de controle do tipo linearizante parcial. As condições propostas permitem garantir a estabilidade robusta do sistema em malha fechada frente a incertezas paramétricas, além de assegurar um certo desempenho com relação a perturbações. Para verificar o comportamento da metodologia proposta, vários testes por simulação são realizados para avaliar o comportamento da estratégia proposta em relação a trabalhos disponíveis na literatura especializada. 
PALAVRAS-CHAVE: bioreatores, cultivo descontínuo com alimentação, realimentação linearizante, controle robusto, LMIs.

\section{INTRODUÇÃO}

A indústria biotecnológica vem crescendo rapidamente, principalmente pelos avanços na compreensão sobre processos biológicos complexos e também pela alta procura dos produtos biologicamente manufaturados como alimentos, bebidas, farmacêuticos, commodities e produtos químicos específicos. O impacto da indústria biotecnológica na economia mundial é substancial como, por exemplo, na produção em grande escala de etanol como combustível renovável (Henson, 2006). Com o avanço da tecnologia de recombinações de DNA, está se tornando cada vez mais comum a criação de cepas de microorganismos especiais para a obtenção de produtos desejados (Stephanopoulos et al., 1998). Tendo em vista que cada célula produz uma quantidade muito pequena desse produto, torna-se necessária uma grande quantidade de células e, com isso, é fundamental que a cepa escolhida tenha um baixo custo, tornando-se viável a produção industrial em grande escala. Entre os microorganismos de baixo custo e de fácil manipulação, destacam-se a bactéria Escherichia Coli e a levedura Saccharomyces Cerevisiae (Tortora et al., 2008). A bactéria $E$. Coli é uma das mais utilizadas na indústria biofarmacêutica (Hafidi et al., 2008) e a levedura S. Cerevisiae é muito utilizada como microorganismo "hospedeiro" para a produção e recombinação de proteínas como na produção de insulina para diabetes, produção de vacinas, entre outros produtos (Renard e Vande Wouwer, 2008; Dewasme e Vande Wouwer, 2008).

A cultura de microorganismos, na maioria das vezes, é realizada em ambientes controlados, chamados de bioreatores, visando atingir uma elevada produtividade além de atender à severas normas de segurança ambiental. Um bioreator pode ser definido como um tanque onde acontecem diversas reações biológicas de formas simultâneas em um meio aquoso (Bastin e Dochain, 1990). Geralmente, o bioreator possui um conjunto de sensores e atuadores como ilustrado na Figura 1.

O sistema de controle de um bioreator deve promover uma condição ótima de crescimento para os microorganismos de maneira que esses possam atingir conversão ou produção de produtos biológicos. Além disso, deve-se prevenir a contaminação de outros organismos e assegurar condições homogêneas de operação em relação à temperatura, $\mathrm{pH}$, oxigênio dissolvido, substrato e concentração de produtos (Nagabhushan e Narula, 2007). Dependendo do sentido da vazão da cultura em um bioreator ou do fornecimento de oxigênio, os modos de operação desse processo podem ser classificados como: descontínuo, descontínuo com alimen-

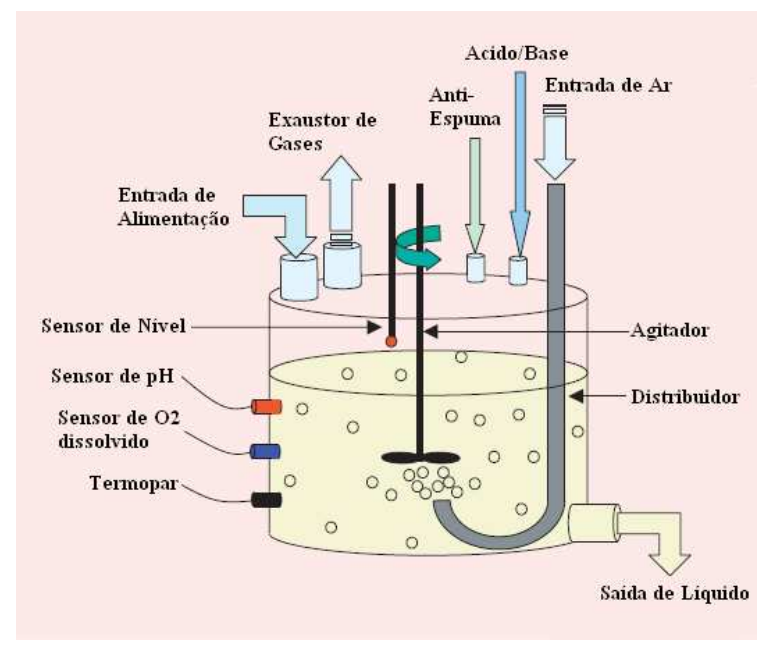

Figura 1: Esquema de um Bioreator (Henson, 2006).

tação e contínuo (Schügerl e Bellgardt, 2000). Em ambientes industriais, os bioreatores operam em regime descontínuo sem alimentação controlada onde, neste caso, o sistema de controle se concentra em manter condições homogêneas de operação. Na última década, a comunidade científica especializada tem proposto vários métodos analíticos avançados para o controle da concentração de substrato e de outras variáveis macroscópicas em bioreatores, visando otimizar a produção do componente desejado (Schügerl e Bellgardt, 2000). As técnicas de produção descontínua com alimentação são o estado da arte em relação à produção eficiente de bactérias e fungos. Processos biológicos modernos com microorganismos geneticamente modificados, geralmente utilizam-se dessa técnica para alcançar altas densidades de células antes de adicionar o agente que iniciará o processo para a obtenção do produto desejado (Nagabhushan e Narula, 2007). Entretanto, duas circunstâncias podem limitar o crescimento em um cultivo descontínuo com alimentação: o acúmulo de produtos inibidores na solução e a falta de volume para a adição de substrato. No cultivo descontínuo com alimentação, nutrientes alimentam continuamente o meio de cultura no bioreator, mas sem que a bimossa seja extraída. Isso significa que o volume do bioreator cresce durante o processo. Este modo de operação é utilizado quando é necessário manter uma concentração de substrato baixa, para otimizar o crescimento ou o produto resultante da fermentação. A principal vantagem é a possibilidade de implementar técnicas que possam controlar as reações químicas através do controle da alimentação injetada no bioreator.

Ao longo dos últimos vinte anos, diversos trabalhos foram propostos na literatura para o controle de bioreatores operando em modo descontínuo com alimentação como, por exemplo, em (Chen et al., 1995), (Van Impe e Bastin, 1995), (Smets et al., 2004), (Bastin e Van Impe, 1995), (El Mouba- 
raki et al., 1993) e (Coutinho e Vande Wouwer, 2010). Nesses trabalhos diversas estratégias de controle foram aplicadas visando determinar um perfil ótimo de alimentação. Esse perfil, por sua vez, é geralmente considerado a única variável manipulada para o controle das variáveis macroscópicas do bioreator. Com o intuito de manter o sistema próximo às condições ótimas de operação em relação ao crescimento da biomassa, pode-se controlar o coeficiente respiratório das células utilizando um analisador de gases (para manter o sistema em um nível crítico de concentração de substrato) ou controlar a concentração do produto secundário para que permaneça em uma pequena quantidade de tal forma que a única reação possível seja a da produção de biomassa. Em particular, considerando a bactéria $E$. Coli como microorganismo de interesse, a técnica de controle preditivo baseada em modelo foi aplicada em Hafidi et al. (2007) e Hafidi et al. (2008); em Roeva e Tzonkov (2005), aplicam-se técnicas de controle ótimo para a determinação da taxa de alimentação que maximiza a produção de biomassa; Jenzch et al. (2006) propõem um controle do tipo GMC (generic model control) para que a variável controlada siga um perfil de alimentação ótimo; e da Rocha (2003) utiliza um controle adaptativo linearizante para a regulação da concentração de acetato. No caso da levedura $S$. Cerevisiae, destacam-se os trabalhos de Dewasme e Vande Wouwer (2008) e Dewasme et al. (2009) que propõem algoritmos de otimização em tempo real para regular o nível de concentração de substrato ao redor do ponto crítico de operação. Entretanto, neste caso, torna-se necessário o conhecimento em tempo real do valor crítico de substrato, o que é geralmente difícil de obter pelos baixos níveis de concentração encontrados nos dois microorganismos. Grande parte das técnicas acima citadas utiliza, de alguma forma, a idéia de realimentação linearizante para definir a estrutura da lei de controle, onde são aplicadas as mais diversas estratégias adicionais de controle para assegurar uma certa robustez à lei de controle proposta (Chen et al., 1995; Coutinho et al., 2009; Renard e Vande Wouwer, 2008).

A realimentação linearizante tem sido utilizada com sucesso na resolução de vários problemas práticos de controle como, por exemplo, no controle de helicópteros, aviões de altaperformance, robôs industriais, dispositivos biomédicos e, em particular, na regulação das variáveis macroscópicas dos bioreatores. Entretanto, esta técnica possui várias deficiências e limitações associadas principalmente à necessidade de um modelo que represente fielmente a dinâmica a ser controlada (Slotine e Li, 1991). Existem duas formas de obtenção da dinâmica linear por realimentação. A primeira delas, denomina-se realimentação entrada-estado (ou input-state linearization) onde toda a representação por variáveis de estado do sistema é linearizada. Na segunda forma, a linearização é feita sob o ponto de vista entrada-saída (input-output linearization) que lineariza o mapeamento entre a entrada $v$ e a saída $y$ do sistema. A linearização por realimentação resulta

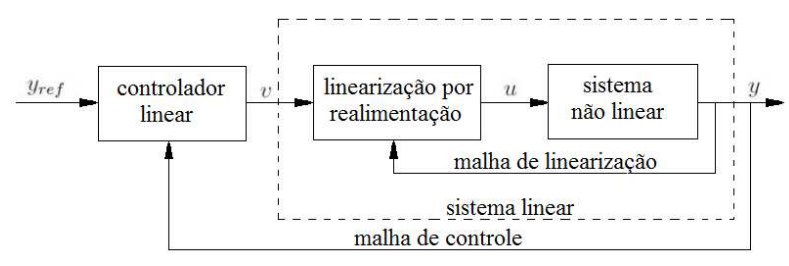

Figura 2: Diagrama em blocos do controle linearizante (Lages, 2009).

em uma dinâmica livre linear. Posteriormente, um controlador linear mais externo é utilizado para atender os requisitos de projeto como ilustrado na Figura 2. Pode-se dizer que o principal problema nessa técnica de controle é a necessidade do cancelamento exato das não-linearidades e, portanto, torna-se necessário um perfeito conhecimento das dinâmicas do sistema. Outro problema relacionado à realimentação linearizante diz respeito à implementação da lei de controle, pois todos os estados que fazem parte da dinâmica não linear devem ser medidos ou mesmo estimados. Além disso, no caso particular da realimentação entrada-saída, parte da dinâmica do sistema não é linearizada. Essa parte não-linear não aparece na dinâmica entre $v$ e $y$ e, portanto, é não controlável. Logo, deve-se verificar se essa dinâmica (chamada de dinâmica zero) é assintoticamente estável antes da implementação da lei de controle. Se o sistema não linear é incerto (que é o caso dos modelos disponíveis para bioreatores), a realimentação linearizante pode levar a um baixo desempenho ou até mesmo a instabilidade do sistema em malha-fechada pelo não cancelamento exato de certas não linearidades do sistema (Henson e Seborg, 1997). Como uma forma de evitar (ou mesmo atenuar) alguns dos problemas relacionados acima, pode-se utilizar uma realimentação linearizante parcial, onde nem todas as não linearidades são canceladas, com os seguintes objetivos: $(i)$ aumentar a robustez da lei de controle; e (ii) diminuir o número de estados a serem utilizados na implementação da realimentação. Ressalta-se que a realimentação parcial, onde algumas não-linearidades presentes na dinâmica original do sistema não são canceladas, pode melhorar o desempenho da lei de controle tanto em termos da resposta transitória quanto da robustez da lei de controle (Freeman e Kokotović, 1997).

Recentemente, em Rohr et al. (2009) foi proposto uma técnica para assegurar uma certa robustez à lei de controle linearizante para uma classe de sistemas não lineares através da escolha adequada da dinâmica livre que resulta da realimentação linearizante. Em resumo, esse trabalho utiliza uma função quadrática e uma descrição das condições de estabilidade em termos de desigualdades matriciais lineares (ou LMIs) dependentes dos estados para garantir a estabilidade robusta do sistema de controle frente a incertezas paramétricas. Ressalta-se que a utilização de LMIs para a formu- 
lação de problemas de controle começou a se desenvolver a partir do final da década de 80 , com a criação e aperfeiçoamento de algoritmos de otimização convexa de pontos interiores. A partir de então, muitos dos resultados usuais da teoria de controle e sistemas, foram reescritos na forma LMI (Boyd et al., 1994; Apkarian et al., 1995). De maneira simplificada, a técnica LMI consiste em reformular as condições de estabilidade por Lyapunov em termos de desigualdades matriciais, onde as restrições de desigualdade devem envolver matrizes simétricas afins nas variáveis de decisão (Coutinho, 2003). Vários problemas complexos já foram formulados por LMIs como análise de estabilidade e síntese de controladores para sistemas lineares a parâmetros variantes, otimização de restrições integrais quadráticas (IQCs), sistemas impulsivos, ganhos variáveis (gain-scheduling) e controle multi-objetivo (El Ghaoui e Niculescu, 2000). Entretanto, a aplicação direta de técnicas LMIs em sistemas não-lineares resulta em desigualdades matriciais não-lineares - ou NLMIs - que não possuem propriedades importantes do ponto de vista de resolução numérica como a convexidade. Mais recentemente, vários trabalhos foram propostos na literatura de controle para contornar este problema onde se destacam as representações por frações lineares - LFR (El Ghaoui e Scorletti, 1996), a formulação por soma de formas quadráticas - SOS (Papachristodoulou e Prajna, 2005), e a representação algébrica-diferencial - DAR (Trofino, 2000; Coutinho et al., 2002; Coutinho e Trofino, 2002). Uma alternativa para utilizar a formulação LMI em sistemas não lineares é modelar as não linearidades do sistema como parâmetros variantes no tempo, que é conhecida na literatura de controle como representação quasi-LPV (Huang e Jadbabaie, 1999). Basicamente, um sistema não-linear

$$
\dot{x}=f(x), x \in \mathcal{X}
$$

é descrito por um sistema dependente de parâmetros

$$
\dot{x}=A(\sigma(x)) x, \sigma(x) \in \Sigma_{x}
$$

$\sigma(x)$ é um vetor de parâmetros contendo as não linearidades do sistema original com limites restritos a um conjunto convexo $\Sigma_{x}$. Todavia, a aproximação quasi- $L P V$ pode conduzir a um elevado grau de conservadorismo e como as condições obtidas são válidas localmente é geralmente necessário estimar uma região invariante no espaço de estados.

Este artigo concentra-se no problema de controle associado à maximização da produção de biomassa considerando os microorganismos E. Coli e S. Cerevisiae. Primeiramente, apresenta-se um modelo geral que descreve a dinâmica do crescimento desses dois microorganismos em bioreatores operando em modo descontínuo com alimentação. Devido à complexidade do modelo (descrito por equações diferenciais não lineares e sujeitas a grandes variações paramétricas), propõe-se uma lei de controle do tipo realimentação linearizante parcial onde a dinâmica livre é escolhida de maneira a assegurar a estabilidade robusta com um certo desempenho frente a sinais de perturbação. Nesta proposta, as taxas de reação de crescimento são modeladas como parâmetros variantes no tempo com valores médios e limites conhecidos levando a uma lei de controle robusta dentro de uma certa região do espaço de estados. Visando melhorar a resposta transitória do sistema, propõe-se também um mecanismo de estimação paramétrica para implementar uma lei de controle dependente de parâmetros (gain scheduling), onde uma função saturação é utilizada para impor os mesmos limites de operação dos parâmetros variantes assegurando, desta forma, a estabilidade do sistema de controle com um sinal de realimentação dependente de parâmetros. Frente à discussão acima, os problemas de controle específicos abordados neste artigo são:

- utilizar uma representação dinâmica que descreva de modo geral o crescimento dos microorganismos E. Coli e S. Cerevisiae;

- projetar uma lei de controle robusta que vise à maximização da reprodução dos microorganismos (produção de biomassa) e que utilize um número reduzido de medições de variáveis biológicas; e

- propor um mecanismo de estimação de parâmetros para melhorar o desempenho da lei de controle robusta, em particular, da taxa de oxidação da glicose.

$\mathrm{O}$ restante do artigo é dividido da seguinte maneira. $\mathrm{Na}$ Seção 2, apresenta-se o modelo dinâmico do bioreator em modo descontínuo com alimentação. Também, discute-se o princípio do bottle-neck que relaciona o ponto de operação ótimo (em termos de maximização da biomassa) com relação à concentração do produto secundário (by-product) formalizando o problema de controle a ser abordado. $\mathrm{Na}$ Seção 3, desenvolve-se uma técnica de controle baseada em uma realimentação linearizante parcial e a representação quasi-LPV, além de propor um mecanismo de adaptação paramétrica para implementar uma lei de controle do tipo gainscheduling. Na sequência, a Seção 4 apresenta resultados de simulação da lei de controle proposta, considerando dois bioreatores disponíveis na literatura de controle, onde são desenvolvidas as duas culturas de microorganismos abordadas neste artigo. Finalmente, a Seção 5 apresenta alguns comentários finais, além de propor possíveis temas de trabalhos futuros.

Notação: $\mathbb{R}^{n}$ denota o conjunto dos vetores reais de dimensão $n, \mathbb{R}^{n \times m}$ denota o conjunto das matrizes reais de ordem $n \times m$ e $I_{n}$ é uma matriz identidade de dimensões $n \times n$. Para matizes reais $S, S^{\prime}$ denota sua transposta e $S>0$ indica que $S$ é positiva definida. 


\section{MODELO DINÂMICO DO BIOREATOR}

O comportamento dinâmico das variáveis biológicas em um bioreator descontínuo com alimentação pode ser descrito da seguinte forma (Hafidi et al., 2007):

$$
\dot{x}=K \varphi(x)-D x-Q_{g a s}(x)+F
$$

sendo $K \in \mathbb{R}^{n \times m}$ uma matriz constante, $\mathrm{e}$

$$
\begin{aligned}
x & =\left[\begin{array}{llll}
x_{1} & x_{2} & \cdots & x_{n}
\end{array}\right]^{\prime} \\
\varphi & =\left[\begin{array}{llll}
\varphi_{1} & \varphi_{2} & \cdots & \varphi_{m}
\end{array}\right]^{\prime} \\
Q_{g a s} & =\left[\begin{array}{llll}
Q_{\text {gas }_{1}} & Q_{\text {gas }_{2}} & \cdots & Q_{\text {gas }_{n}}
\end{array}\right]^{\prime} \\
F & =\left[\begin{array}{llll}
F_{1} & F_{2} & \cdots & F_{n}
\end{array}\right]^{\prime}
\end{aligned}
$$

No modelo acima: $x$ representa o vetor de estados cujos elementos são as concentrações das várias espécies dentro do meio aquoso; o termo $K \varphi(x)$ descreve a cinética das reações microbiológicas e bioquímicas envolvidas no processo; e os termos restantes $-D x-Q_{\text {gas }}(x)+F$ descrevem os transportes dos componentes para dentro e fora do bioreator. A matriz $K$ é formada pelas constantes pseudo-estequiométricas $k_{i}$, a função vetorial $\varphi(x)$ representa as taxas de crescimento não linear relacionadas às reações existentes, $D$ é a taxa de diluição, $Q_{\text {gas }}$ representa a taxa de transferência da fase líquida para a gasosa e $F$ é a alimentação do bioreator.

Dentro de um bioreator existem diversas reações simultâneas que são modeladas por equações não lineares e envolvem variáveis macroscópicas, sendo essas obtidas através da análise empírica das concentrações das substâncias encontradas na cultura de microorganismos (Bastin e Dochain, 1990). A seguir, apresentam-se as principais reações existentes tanto no metabolismo da bactéria $E$. Coli quanto da levedura S. Cerevisiae (Hafidi et al., 2007; Bastin e Dochain, 1990; Dewasme et al., 2009):

- Oxidação da glicose

$$
k_{1} S+k_{2} O \stackrel{\varphi_{1}}{\longrightarrow} k_{3} X+k_{4} C
$$

- Fermentação da glicose

$$
k_{5} S+k_{6} O \stackrel{\varphi_{2}}{\longrightarrow} k_{7} X+k_{8} C+k_{9} P
$$

- Oxidação do produto secundário

$$
k_{10} P+k_{11} O \stackrel{\varphi_{3}}{\longrightarrow} k_{12} X+k_{13} C
$$

sendo que $X, S, P, O$ e $C$ representam respectivamente as concentrações de biomassa, substrato (glicose), produto secundário, oxigênio e dióxido de carbono. As constantes $k_{i}$ são os coeficientes pseudo-estequiométricos. O produto secundário $P$ resultante da cultura de $E$. Coli é o ácido acético e da cultura de $S$. Cerevisiae é o etanol.

As taxas de crescimento definidas pelas funções $\varphi_{i}, i=$ $1,2,3$, são dadas por:

$$
\varphi_{i}=r_{i} X, i=1,2,3
$$

$r_{i}$ são as taxas de crescimento específico que dependem do regime de operação da cultura e, portanto, são modeladas por funções descontínuas. Neste trabalho, consideramse os seguintes modelos baseados no princípio do bottleneck (Sonnleitner e Käppeli, 1986):

- Taxa da reação de respiração da glicose

$$
r_{1}=\min \left(r_{S}, r_{\text {Scrit }}\right)
$$

- Taxa da reação de fermentação da glicose

$$
r_{2}=\max \left(0, r_{S}-r_{\text {Scrit }}\right)
$$

- Taxa da reação de oxidação do produto secundário

$$
r_{3}=\frac{\max \left(0, \min \left(r_{P}, \frac{k_{o s}\left(r_{S c r i t}-r_{S}\right)}{k_{o a}}\right)\right)}{k_{11}}
$$

$r_{S}$ é associado à cinética do consumo de substrato, $r_{S c r i t}$ representa o consumo crítico de substrato, que geralmente depende da capacidade respiratória $r_{O}$ e da taxa de oxidação do produto $r_{P}$. Essas taxas são definidas com base na clássica lei de Monod (Bastin e Dochain, 1990) e são dadas por:

$$
\begin{gathered}
r_{S}=\frac{\mu_{S}}{k_{1}} \frac{S}{S+K_{s}} \\
r_{S c r i t}=\frac{r_{O}}{k_{1}}, r_{O}=\frac{\mu_{O}}{k_{2}} \frac{O}{K_{O}+O} \frac{K_{i, P}}{K_{i, P}+P} \\
r_{P}=\mu_{P} \frac{P}{K_{P}+P}
\end{gathered}
$$

$\mu_{S}, \mu_{O}$ e $\mu_{P}$ são os valores máximos das respectivas taxas de crescimento específico; $K_{S}, K_{O}$ e $K_{P}$ são as constantes de saturação dos elementos correspondentes e $K_{i, P}$ é a constante de inibição. As constantes $k_{o s}$ e $k_{o a}$ representam os coeficientes que caracterizam os produtos entre o consumo de oxigênio $\times$ substrato e produto entre o consumo de oxigênio $\times$ produto secundário.

A seguir, apresentam-se as equações diferenciais que descrevem o comportamento das dinâmicas das variáveis macroscópicas no bioreator obtidas através do balanceamento de massa (Dewasme et al., 2010): 
- Concentração de Biomassa

$$
\frac{d X}{d t}=\left(k_{1} r_{1}+k_{2} r_{2}+k_{3} r_{3}\right) X-D X
$$

- Concentração de Substrato

$$
\begin{aligned}
\frac{d S}{d t} & =\left(k_{4} r_{1}+k_{5} r_{2}+k_{6} r_{3}\right) X-D S \\
& +S_{i n} D
\end{aligned}
$$

- Concentração do Produto Secundário

$$
\frac{d P}{d t}=\left(k_{7} r_{1}+k_{8} r_{2}+k_{9} r_{3}\right) X-D P
$$

- Concentração de Oxigênio

$$
\begin{aligned}
\frac{d O}{d t} & =\left(k_{10} r_{1}+k_{11} r_{2}+k_{12} r_{3}\right) X-D O \\
& +K_{L a}\left(O_{\text {sat }}-O\right)
\end{aligned}
$$

- Concentração de Dióxido de Carbono

$$
\begin{aligned}
\frac{d C}{d t} & =\left(k_{13} r_{1}+k_{14} r_{2}+k_{15} r_{3}\right) X-D C \\
& +K_{L a}\left(C_{s a t}-C\right)
\end{aligned}
$$

$S_{i n}$ é a concentração do substrato de entrada, $D$ é a taxa de diluição que é dada pela relação entre a vazão de entrada no bioreator $F_{i n}$ e o volume da cultura no bioreator $V_{B}$. Os termos $k_{L a}\left(O_{\text {sat }}-O\right)$ e $k_{L a}\left(C_{s a t}-C\right)$ representam as taxas de mudança de fase gasosa para a líquida do oxigênio e do dióxido de carbono, respectivamente, sendo $O_{\text {sat }}$ e $C_{\text {sat }}$ as concentrações em saturação de oxigênio e do dióxido de carbono.

Observação $1 \mathrm{O}$ volume da cultura no bioreator, representado por $V_{B}$, não aparece explicitamente no modelo dinâmico do bioreator, pois a taxa de diluição (dada por $D=$ $F_{i n} / V_{B}$ ) é considerada como sinal de controle. Assume-se que existe um dispositivo adicional de controle que indica que o volume da cultura atingiu o volume máximo do bioreator.

\section{Princípio do Bottle-neck}

Os modelos das taxas de crescimento específico definidas em (6)-(8) são baseados na suposição de Sonnleitner conhecida como princípio do bottle-neck (Sonnleitner e Käppeli, 1986), que foi inicialmente aplicado à levedura $S$. Cerevisiae. Este princípio demonstra que durante a cultura de microorganismos, as células provavelmente mudam seu metabolismo devido à limitação na capacidade de oxidação. Além disso, o
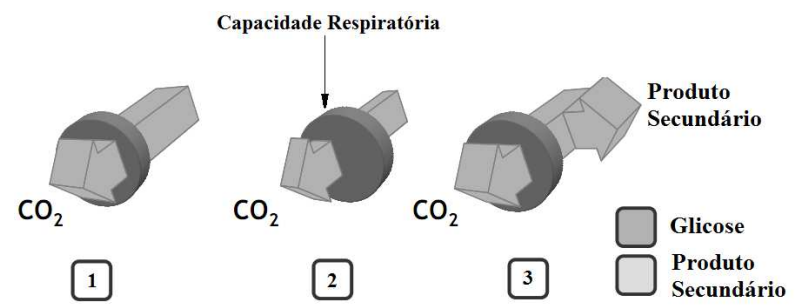

Figura 3: Regimes de operação da cultura pelo princípio do bottle-neck: (1) ótimo, (2) respiratório e (3) respirofermentativo.

princípio do bottle-neck permite estabelecer as condições necessárias para se obter as condições ótimas de operação.

Basicamente, este princípio estabelece que a glicose tem dois caminhos de degradação ao produzir o etanol (no caso da levedura $S$. Cerevisiae) na presença de oxigênio como ilustrado na Figura 3. Como durante o processo no interior do bioreator as células modificam seu metabolismo em relação ao limite de capacidade respiratória, parte é consumido/metabolizado pela oxidação e a outra parte é reduzida, sendo o etanol o produto secundário $(P)$ da energia do metabolismo reduzido. Quando o substrato é excedente (concentração de $S>S_{\text {crit }}$ e taxa de consumo de glicose $r_{s}>r_{\text {Scrit }}$ ), as células produzem um produto do metabolismo $P$ através da fermentação, e a cultura é dita estar em regime respiro-fermentativo (RF). Quando o substrato é o limitante (concentração de $S<S_{\text {crit }}$ e taxa de consumo de glicose $r_{s}<r_{\text {Scrit }}$ ), o substrato disponível, geralmente a glicose, e o produto metabolizado $P$, se presente no meio, é oxidado. Neste caso, diz-se que a cultura se encontra em regime respiratório $(\mathbf{R})$.

Para a obtenção da máxima produtividade, é necessário que se trabalhe entre os limites dos regimes respiro-fermentativo e respiratório. É possível fazer o controle desses estados utilizando o valor da concentração do produto $P$, que deve ter um valor próximo a zero para que o processo fique entre faixas RF e $\mathbf{R}$ de operação.

A condição ótima de operação (em termos de produção de biomassa) é atingida quando o nível de concentração de $S$ é igual ao seu valor crítico, isto é:

$$
r_{1}=r_{S}=r_{S c r i t} \text { e } r_{2}=0
$$

A partir das igualdades acima, pode-se facilmente deduzir a expressão do valor ótimo da concentração de substrato:

$$
S_{c r i t}=\frac{r_{O} K_{S}}{\mu_{S}-K_{S}}
$$

Uma possível estratégia de controle é regular a concentração de substrato no seu valor crítico e desta forma operar no 
regime ótimo para a maximização da biomassa como, por exemplo, adotado em (Coutinho et al., 2009). No entanto, esta estratégia exige a estimação da taxa de capacidade respiratória $r_{O}$ a partir da medição online das concentrações de $O, P$ e $S$ e do conhecimento exato das constantes envolvidas que são altamente incertas. Neste artigo, utiliza-se uma técnica alternativa na qual se regula o nível de concentração do produto secundário $P$ em valores próximos a zero ${ }^{1}$.

\section{PROJETO DO CONTROLADOR}

Nesta seção, propõe-se uma técnica de controle visando regular o valor da concentração do produto $P$ em valores próximos a zero definido pelo valor de referência $P^{*}$. Desta forma, o metabolismo do microorganismo é obrigado a operar próximo a sua condição ótima (i.e., $S \approx S_{\text {crit }}$ ). Com este objetivo, utiliza-se como variável manipulada o fluxo de alimentação $F_{i n}$ ou, equivalentemente, a taxa de diluição $D$.

Inicialmente, consideram-se as seguintes suposições simplificadoras no sistema de controle do bioreator como feito na maioria das referências dedicadas à maximização da biomassa em bioreatores: $(i)$ existe um controlador específico para manter o $\mathrm{pH}$ e a temperatura nos níveis ideais de operação; e (ii) existe um sistema supervisório que controla a exaustão de gases, a aeração, o misturador e que indica a aproximação do nível máximo visando impedir que exista vazamento do bioreator.

Para uma melhor compreensão da lei de controle a ser proposta, apresenta-se a seguir uma representação no espaço de estados da dinâmica descrita em (12)-(16):

$$
\dot{x}=K r(x) x_{1}+A x-\left(x-B_{u}\right) u+E
$$

$x=\left[\begin{array}{lllll}x_{1} & x_{2} & x_{3} & x_{4} & x_{5}\end{array}\right]^{\prime}=\left[\begin{array}{lllll}X & S & P & O & C\end{array}\right]^{\prime}$ é o vetor de estados, $r(x)=\left[\begin{array}{lll}r_{1} & r_{2} & r_{3}\end{array}\right]^{\prime}$ é o vetor de taxas de crescimento específicas, e $u=D=F_{i n} / V_{B}$ é a entrada de controle (representando a taxa de diluição). Neste modelo, as matrizes $K$ e $A$ e os vetor $B_{u}, E$ são dados por:

$$
\begin{aligned}
K & =\left[\begin{array}{ccc}
k_{1} & k_{2} & k_{3} \\
k_{4} & k_{5} & k_{6} \\
k_{7} & k_{8} & k_{9} \\
k_{10} & k_{11} & k_{12} \\
k_{13} & k_{14} & k_{15}
\end{array}\right], B_{u}=\left[\begin{array}{c}
0 \\
S_{i n} \\
0 \\
0 \\
0
\end{array}\right], \\
A & =\left[\begin{array}{ccc}
0_{3 \times 2} & 0_{3 \times 2} & 0_{3 \times 1} \\
0_{2 \times 2} & -k_{L a} I_{2 \times 2} & 0_{2 \times 2}
\end{array}\right], \\
E & =\left[\begin{array}{lllll}
0 & 0 & 0 & k_{L a} O_{\text {sat }} & k_{L a} C_{\text {sat }}
\end{array}\right]^{\prime}
\end{aligned}
$$

A partir deste ponto a concentração da biomassa é representada pelo estado $x_{1}$, a concentração do substrato é represen-

\footnotetext{
${ }^{1} \mathrm{Na}$ realidade, em valores baixos de acordo com a sensibilidade do sensor do produto $P$.
}

tada pelo estado $x_{2}$, a concentração do produto secundário por $x_{3}$, a concentração do oxigênio por $x_{4}$ e a concentração do dióxido de carbono por $x_{5}$.

\section{Linearização Parcial}

Para a obtenção da lei de controle que regula o valor de $x_{3}$ no valor de referência $x_{3}^{*}$, utiliza-se a técnica de realimentação linearizante parcial. Primeiramente, assume-se que: $(i)$ o valor teórico de $S_{\text {crit }}$ é muito pequeno (tipicamente abaixo de $0,1 \mathrm{~g} / \mathrm{l})$; ( $i i)$ não existe acúmulo de glicose no momento da operação do bioreator, nem nas vizinhanças do ponto de operação; (iii) a pequena quantidade de substrato é instantaneamente consumida pelas células. Desta forma, pode-se supor que $\frac{d x_{2}}{d t} \approx 0$ e $x_{2} \approx 0$. Utilizando a equação da dinâmica do substrato (13) aqui representada novamente como (20), considere os seguintes passos:

$$
\dot{x}_{2}=-k_{1} x_{1}-r_{2} x_{1}-\left(x_{2}-S_{i n}\right) u
$$

utilizando as suposições $\frac{d x_{2}}{d t} \approx 0$ e $x_{2} \approx 0$ na expressão acima, chega-se a:

$$
-k_{1} x_{1}-r_{2} x_{1}-S_{i n} u \approx 0 \text { e } r_{2} x_{1} \approx-r_{1} x_{1}+S_{i n} u
$$

Na equação acima é obtida a expressão de $r_{2} x_{1}$ para que atue entre o regime respiro-fermentativo e respiratório (situação ótima). Agora, considerando a equação da dinâmica do produto secundário (14), aqui novamente representada pela equação (22):

$$
\dot{x}_{3}=\left(k_{8} r_{2}+k_{9} r_{3}\right) x_{1}-u x_{3}
$$

e substituindo pela equação (21), chega-se à seguinte expressão:

$$
\dot{x}_{3}=k_{8}\left(-r_{1} x_{1}+S_{\text {in }} u\right)-r_{3} x_{1}-x_{3} u
$$

que pode ser simplificada. Logo, torna-se possível reescrever a derivada de $x_{3}$ como:

$$
\dot{x_{3}}=-\left(k_{8} r_{1}+r_{3}\right) x_{1}+\left(k_{8} S_{i n}-x_{3}\right) u
$$

Em (Coutinho e Vande Wouwer, 2010), utiliza-se uma realimentação linearizante completa para obter uma dinâmica linear. Esta lei de controle necessita da estimativa das taxas de reação específicas $r_{1}$ e $r_{3}$. Neste artigo, implementa-se uma realimentação parcial na qual se despreza o termo $r_{3}$, pois na condição ótima de operação $r_{3} \approx 0$ e a taxa $r_{1}$ é modelada como um parâmetro variante no tempo:

$$
r_{1}(x)=r_{1}(t)=\bar{r}_{1}+\sigma(t) \hat{r}_{1}, \sigma(t) \in[-1,1]
$$

$\bar{r}_{1}$ representa o valor médio assumido pela taxa de crescimento específico $r_{1}(t)$ e o termo $\sigma(t) \hat{r}_{1}$ é o desvio do valor médio em relação ao valor real $r_{1}(t)$. Assumindo que o valor 
máximo $\left(r_{1_{\max }}\right)$ e o valor mínimo $\left(r_{1_{\text {min }}}\right)$ de $r_{1}$ são conhecidos, os valores de $\bar{r}_{1}$ e $\hat{r}_{1}$ são obtidos através das seguintes expressões:

$$
\bar{r}_{1}=\frac{r_{1_{\max }}+r_{1_{\min }}}{2}, \hat{r}_{1}=\frac{r_{1_{\max }}-r_{1_{\min }}}{2}
$$

Observação 2 As variáveis macroscópicas em um bioreator, que são os estados do sistema dinâmico (18), têm valores máximo e mínimo conhecidos com base em resultados experimentais que são utilizados na identificação dos parâmetros do modelo. Esta informação pode ser utilizada para obter uma estimativa dos valores de $\bar{r}_{1}$ e $\hat{r}_{1}$ em (25).

A partir das simplificações acima, projeta-se uma realimentação linearizante considerando apenas o valor médio de $r_{1}$ levando a seguinte lei de controle (linearização parcial):

$$
u_{L P}=\frac{1}{k_{8} S_{i n}-x_{3}}\left(k_{8} \bar{r}_{1} x_{1}+\lambda\left(x_{3}^{*}-x_{3}\right)\right)
$$

$\lambda\left(x_{3}^{*}-x_{3}\right)$ representa a dinâmica linear arbitrária a ser imposta pela lei linearizante, e $x_{3}^{*}$ é o valor desejado para a concentração do produto secundário.

Observação 3 Condições próximas à região ótima de operação podem ser atingidas fazendo com que o produto secundário seja pequeno ou aproximadamente igual a zero. A lei de controle proposta em (26) leva (assintoticamente) o valor de $x_{3}$ ao valor de referência $x_{3}^{*}$ que é escolhido tão próximo quanto possível de zero dependendo da sensibilidade do sensor do produto secundário (i.e., o estado $x_{3}$ ).

\section{Determinação do Parâmetro $\lambda$}

Neste trabalho, determina-se o valor do parâmetro $\lambda$, que define a dinâmica livre imposta pela lei de controle em (26), de maneira a assegurar uma certa robustez à lei de controle linearizante e também para obter um certo desempenho frente a perturbações externas. Com este objetivo, utiliza-se a formulação LMI para descrever um problema de otimização que minimiza a norma $\mathcal{H}_{\infty}$ do modelo quasi-LPV do sistema resultante em malha fechada.

Relembrando, a definição do parâmetro variante no tempo $\sigma=\sigma(t)$, utilizada para modelar a taxa de crescimento $r_{1}=$ $r_{1}(x)$ em (24), representa o termo não linear

$$
\frac{r_{1}(x)-\bar{r}_{1}}{\hat{r}_{1}}
$$

Aplicando-se a lei de controle em (26) no subsistema da dinâmica de $x_{3}$ em (23), obtém-se:

$$
\dot{x_{3}}=-\lambda x_{3}+\lambda x_{3}^{*}-r_{3} x_{1}+k_{8}\left(\overline{r_{1}}-r_{1}\right) x_{1}
$$

Assumindo que $r_{3} \approx 0$, chega-se à seguinte expressão para a dinâmica do produto secundário:

$$
\dot{x_{3}}=\lambda\left(x_{3}^{*}-x_{3}\right)+k_{8}\left(\overline{r_{1}}-r_{1}\right) x_{1}
$$

Definindo $\xi=x_{3}^{*}-x_{3}$, pode-se representar a dinâmica acima através do seguinte mapeamento entrada-saída:

$$
\mathcal{M}:\left\{\begin{array}{c}
\dot{\xi}=-\lambda \xi-\hat{r}_{1} \sigma(t) w \\
z=\xi
\end{array}\right.
$$

$w:=k_{8} x_{1}$ é visto como uma perturbação externa ao modelo $\mathcal{M}, \sigma(t) \in \Delta:=[-1,1]$, e $z$ representa a variável de desempenho (i.e., o erro de seguimento).

O parâmetro $\lambda$ é projetado para atenuar o efeito do termo não-linear que não foi cancelado, representado por $\hat{r}_{1} \sigma w$. Com este objetivo, utiliza-se um controle do tipo $\mathcal{H}_{\infty}$ para a obtenção do valor de $\lambda$, como detalhado a seguir.

Considerando um intervalo de tempo com horizonte finito (de 0 a $T$ ), pode-se definir o ganho- $\mathcal{L}_{2}$ do sistema na seguinte maneira supondo condições iniciais nulas:

$$
\left\|\mathcal{M}_{w z}\right\|_{\infty,[0, T]}:=\sup _{\sigma \in \Delta, 0 \neq w} \frac{\|z\|_{2,[0, T]}}{\|w\|_{2,[0, T]}}
$$

sendo $T$ tipicamente o tempo de produção da biomassa (uma batelada). Assume-se que $w$ é um sinal com energia finita no horizonte de tempo $T$ (i.e., $w \subset \mathcal{L}_{2,[0, T]}$ ).

Um limitante superior $\alpha$ de $\left\|\mathcal{M}_{w z}\right\|_{\infty,[0, T]}$ pode ser minimizado através do seguinte problema de otimização

$$
\min _{\lambda, \alpha} \alpha:\left\|\mathcal{M}_{w z}\right\|_{\infty,[0, T]} \leq \alpha, \sigma \in \Delta
$$

$\lambda$ e $\alpha$ são as variáveis de decisão. Note que o problema de otimização acima definido visa minimizar o ganho- $\mathcal{L}_{2,[0, T]} \mathrm{e}$ ao mesmo tempo garantir a estabilidade robusta do sistema (28).

Para a resolução numérica do problema de otimização em (30), utiliza-se a teoria de estabilidade de Lyapunov e uma descrição das condições de estabilidade em termos de LMIs. Com este objetivo, considere a seguinte função de Lyapunov Quadrática:

$$
V(\xi)=\xi^{\prime} Q \xi=Q \xi^{2}
$$

$Q$ é um escalar positivo a ser determinado.

Para assegurar a estabilidade do sistema com perturbação, impõe-se a seguinte restrição da derivada da função de Lyapunov (Boyd et al., 1994):

$$
\min \alpha: V(\xi)>0, \dot{V}(\xi)+\frac{1}{\alpha} z^{\prime} z-\alpha w^{\prime} w<0
$$


Para determinar $\dot{V}(\xi)$ é necessário considerar a dinâmica de $\xi$, descrita pela equação (28), levando a seguinte expressão:

$$
\begin{aligned}
\dot{V}(\xi) & =2 \xi Q \dot{\xi}=2 \xi Q\left(-\lambda \xi+\hat{r}_{1} \sigma w\right) \\
& =\left[\begin{array}{c}
\xi \\
w
\end{array}\right]^{\prime}\left[\begin{array}{cc}
-2 Q \lambda & \hat{r}_{1} Q \sigma \\
\hat{r}_{1} \sigma Q & 0
\end{array}\right]\left[\begin{array}{c}
\xi \\
w
\end{array}\right]
\end{aligned}
$$

Utilizando a equação (33) em (32), chega-se à seguinte desigualdade:

$$
\left[\begin{array}{c}
\xi \\
w
\end{array}\right]^{\prime}\left[\begin{array}{cc}
-2 m & \hat{r}_{1} Q \sigma \\
\hat{r}_{1} \sigma Q & -\alpha
\end{array}\right]\left[\begin{array}{c}
\xi \\
w
\end{array}\right]-\frac{1}{\alpha} z^{\prime} z<0
$$

$m=\lambda Q$ é a parametrização do controlador.

Utilizando o complemento de Schur na expressão acima temse a seguinte condição suficiente para (34):

$$
\min _{Q, m, \alpha} \alpha:\left\{\begin{array}{c}
\alpha>0, Q>0, m>0 \\
{\left[\begin{array}{ccc}
-2 m & \hat{r}_{1} \sigma_{1} Q & 1 \\
\hat{r}_{1} \sigma_{1} Q & -\alpha & 0 \\
1 & 0 & -\alpha
\end{array}\right]<0} \\
{\left[\begin{array}{ccc}
-2 m & \hat{r}_{1} \sigma_{2} Q & 1 \\
\hat{r}_{1} \sigma_{2} Q & -\alpha & 0 \\
1 & 0 & -\alpha
\end{array}\right]<0}
\end{array}\right.
$$

$\sigma_{1}=-1$ e $\sigma_{2}=1$.

Supondo que exista uma solução para o problema de otimização acima, então o sistema $\mathcal{M}$ é localmente estável e o parâmetro do controlador $\lambda$ é obtido pela relação $\lambda=m Q^{-1}$.

Observação 4 Por simplicidade, neste artigo não se considera nenhuma restrição no valor a ser determinado para o parâmetro $\lambda$ visando a limitação do sinal de controle. Caso seja necessário, pode-se incluir uma restrição na forma de um regulador quadrático ou do tipo saturação como em (Coutinho et al., 2002; Coutinho e Trofino, 2004).

\section{Estimação do Parâmetro $\sigma(t)$}

A lei de controle proposta garante a estabilidade do sistema em malha fechada, mas pode levar a resultados conservadores quando comparamos a resposta transitória em relação à outras referências que consideram uma lei de controle mais complexa. Como visto na equação (26), a realimentação linearizante parcial foi projetada apenas considerando o valor médio da taxa $r_{1}$, que é representado por $\bar{r}_{1}$. Nesta seção, propõe-se um mecanismo simples de estimação do parâmetro variante no tempo

$$
\sigma(t)=\frac{\bar{r}_{1}-r_{1}}{\hat{r}_{1}}
$$

Desta forma, torna-se possível implementar uma lei de controle dependente de parâmetros (gain-scheduling) visando obter uma possível melhora no desempenho do sistema de controle. Este mecanismo de estimação online é bastante simples e é implementado através de uma equação dinâmica de primeira ordem.

O mecanismo de estimação de $r_{1}$ é construído com base na informação do valor de $\xi$ que deve ser próximo a zero quando a estimativa $\tilde{\sigma}(t)$ se aproxima do valor real $\sigma(t)$. Esta lógica de estimação é formulada da seguinte maneira:

$$
\left\{\begin{array}{l}
\dot{\hat{\sigma}}=-\phi_{1} \hat{\sigma}+\phi_{2} \xi \\
\tilde{\sigma}=\operatorname{sat}(\hat{\sigma}) \\
\tilde{r}_{1}=\bar{r}_{1}+\tilde{\sigma} \hat{r}_{1}
\end{array}\right.
$$

$\tilde{\sigma}$ é a estimativa de $\sigma, \phi_{1}$ e $\phi_{2}$ são constantes que definem a dinâmica do mecanismo de estimação paramétrica, $\tilde{r}_{1}$ é a estimativa da taxa de crescimento $r_{1}$, e sat( $\cdot$ ) representa a função saturação unitária. Note que utiliza-se como estimativa de $\sigma$ a função saturação de $\hat{\sigma}$. Desta forma, garante-se que a estimativa de $\sigma$ nunca assumirá um valor fora dos valores admissíveis, isto é, $\tilde{\sigma} \in[-1,1]$. Desta forma, ao se utilizar o mecanismo acima em conjunto com a lei de controle em (26) a estabilidade local da lei de controle é garantida.

Com base na estimativa acima, a lei de controle é redefinida da seguinte maneira:

$$
u_{L P V}=\frac{1}{k_{8} S_{i n}-x_{3}}\left(\left(k_{8} \tilde{r}_{1}\right) x_{1}+\lambda\left(x_{3}^{*}-x_{3}\right)\right)
$$

Observação 5 Uma das grandes vantagens da utilização da lei de controle (37) é o fato da sua implementação apenas necessitar da medição dos estados $x_{1}$ e $x_{3}$ e não sendo necessário medir os estados $x_{2}$ e $x_{4}$. Desta forma, evita-se a utilização de sensores tendo como consequência considerável redução nos custos da implementação prática da lei de controle proposta, tanto pela não utilização de sensores quanto pela não utilização de mecanismos complexos de estimação de estados (soft sensors), como proposto nas referências (Chen et al., 1995; Dewasme e Vande Wouwer, 2008; Coutinho et al., 2009; Renard e Vande Wouwer, 2008; Bastin e Dochain, 1990) que utilizam um número igual ou maior de sensores para o mesmo processo.

\section{RESULTADOS DE SIMULAÇÃO}

Nesta seção são realizados diversos testes em simulação considerando dois bioreatores com volumes distintos (Dewasme et al., 2010). O primeiro, de 5 litros, é utilizado para a cultura da bactéria E. Coli, e o segundo, de 20 litros, é utilizado para a cultura da levedura $S$. Cerevisiae. Primeiramente, simulações em malha aberta são realizadas como o objetivo 
Tabela 1: Condições iniciais e alguns parâmetros para as culturas de E. Coli e S. Cerevisiae.

\begin{tabular}{|c|c|c|c|}
\hline Parâmetro & S. Cerevisiae & E. Coli & Unidade \\
\hline$x_{1}(0)$ & 0,4 & 0,4 & $\mathrm{~g} / \mathrm{l}$ \\
\hline$x_{2}(0)$ & 0,5 & 0,05 & $\mathrm{~g} / \mathrm{l}$ \\
\hline$x_{3}(0)$ & 0,8 & 0,8 & $\mathrm{~g} / \mathrm{l}$ \\
\hline$x_{4}(0)$ & 0,035 & 0,035 & $\mathrm{~g} / \mathrm{l}$ \\
\hline$x_{5}(0)$ & 1,286 & 1,286 & $\mathrm{~g} / \mathrm{l}$ \\
\hline$O_{\text {sat }}$ & 0,035 & 0,035 & $\mathrm{~g} / \mathrm{l}$ \\
\hline$C_{\text {sat }}$ & 1,286 & 1,286 & $\mathrm{~g} / \mathrm{l}$ \\
\hline$V_{B}(0)$ & 6,8 & 3,5 & 1 \\
\hline$S_{\text {in }}$ & 350 & 250 & $\mathrm{~g} / \mathrm{l}$ \\
\hline
\end{tabular}

de estimar os valores máximo e mínimo admissíveis para a taxa de reação $r_{1}$. Posteriormente, determina-se o valor do parâmetro $\lambda$ que define a dinâmica livre imposta pela lei de controle. Em seguida, diversas simulações do sistema em malha fechada, considerando a lei de controle robusta proposta em (26), são realizadas com o objetivo de avaliar o desempenho do controlador. Finalizando os testes por simulação, implementa-se a lei de controle proposta em (37) com o objetivo de avaliar a eficiência do mecanismo de estimação da taxa de alimentação $r_{1}$. Todas as simulações foram realizadas com o auxílio da plataforma Matlab/Simulink ${ }^{\circledR}$.

\section{Parâmetros de Simulação}

A seguir, apresentam-se diversas tabelas com os valores das condições iniciais utilizadas para os dois bioreatores, além dos valores dos parâmetros do modelo descrito em (18) considerando as culturas de E. Coli e S. Cerevisiae.

As condições iniciais utilizadas nas simulações, considerando as duas culturas de microorganismos, são apresentadas na Tabela 1 e foram obtidas da referência (Dewasme et al., 2010). Os parâmetros dos modelos (coeficientes estequiométricos e constantes cinéticas) são apresentados na Tabela 2 para a bactéria $E$. Coli e têm como base o trabalho (da Rocha, 2003). No caso da levedura S. Cerevisiae, esses parâmetros são apresentados na Tabela 3, onde os valores foram obtidos em (Sonnleitner e Käppeli, 1986).

\section{Simulação em Malha Aberta}

Para a simulação do sistema em malha aberta, as equações dinâmicas (12), (13), (14), (15) e (16) foram implementadas através de uma M-file S-function, como ilustrado na Figura 4.

Note que para a implementação da lei de controle proposta em (26) é necessário determinar os valores de $\bar{r}_{1}$ e $\hat{r}_{1}$ a partir dos valores $r_{1_{\text {min }}}$ e $r_{1_{\max }}$. Neste artigo, os valores desses parâmetros foram obtidos através da simulação da resposta
Tabela 2: Parâmetros do modelo para E. Coli.

\begin{tabular}{|c|c|c|}
\hline Coeficientes & Valores & Unidades \\
\hline$k_{1}$ & 1 & - \\
\hline$k_{2}$ & 1 & - \\
\hline$k_{3}$ & 1 & - \\
\hline$k_{4}$ & 0,316 & g de $S / g$ de $X$ \\
\hline$k_{5}$ & 0,04 & g de $S / g$ de $X$ \\
\hline$k_{6}$ & 0 & - \\
\hline$k_{7}$ & 0 & - \\
\hline$k_{8}$ & 0,157 & $g \operatorname{de} P / g \operatorname{de} X$ \\
\hline$k_{9}$ & 0,432 & $g$ de $P / g$ de $X$ \\
\hline$k_{10}$ & 0,339 & $\mathrm{gde} \mathrm{O}_{2} / \mathrm{g}$ de $X$ \\
\hline$k_{11}$ & 0,471 & $g \operatorname{de} \mathrm{O}_{2} / g$ de $X$ \\
\hline$k_{12}$ & 0,955 & $g \mathrm{de} \mathrm{O}_{2} / g \operatorname{de} X$ \\
\hline$k_{13}$ & 0,405 & $\mathrm{~g} \mathrm{de} \mathrm{CO}_{2} / \mathrm{g}$ de $\mathrm{X}$ \\
\hline$k_{14}$ & 0,754 & $\mathrm{~g}$ de $\mathrm{CO}_{2} / \mathrm{g}$ de $\mathrm{X}$ \\
\hline$k_{15}$ & 1,03 & $g$ de $\mathrm{CO}_{2} / g$ de $\mathrm{X}$ \\
\hline$\mu_{O}$ & 0,7218 & $g$ de $O_{2} / g$ de $X / h$ \\
\hline$\mu_{S}$ & 1,832 & $g$ de $S / g$ de $X / h$ \\
\hline$K_{O}$ & 0,0001 & $\mathrm{gde} \mathrm{O}_{2} / \mathrm{l}$ \\
\hline$K_{S}$ & 0,1428 & $g$ de $S / I$ \\
\hline$K_{P}$ & 0,5236 & $g$ de $P / l$ \\
\hline$K_{i_{P}}$ & 6,952 & $g$ de $P / 1$ \\
\hline$k_{o s}$ & 2,02 & $\mathrm{gde} \mathrm{O}_{2} / \mathrm{g} \operatorname{de} X$ \\
\hline$k_{o p}$ & 1,996 & $\mathrm{gde} \mathrm{O}_{2} / \mathrm{g}$ de $X$ \\
\hline
\end{tabular}

livre do sistema em malha aberta. Os resultados obtidos são apresentados nas Figuras 5 e 6, supondo $F_{\text {in }}=0$ e as condições iniciais dadas na Tabela 1. Utilizando a definição da taxa de reação $r_{1}$ como apresentado em (6), chegam-se aos valores apresentados na Tabela 4.

\section{Simulação em Malha Fechada}

Para analisar o desempenho das leis de controle propostas na seção anterior, a lei de controle foi implementada utilizando os blocos disponíveis na plataforma Simulink. Em situações práticas o valor de referência para o produto secundário (que deve ser próximo a zero, i.e., $x_{3}^{*} \approx 0$ ) é definido com base

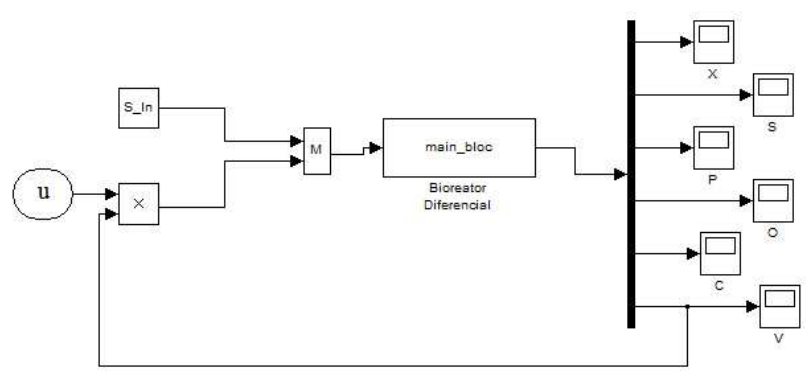

Figura 4: Diagrama em malha aberta no Simulink. 

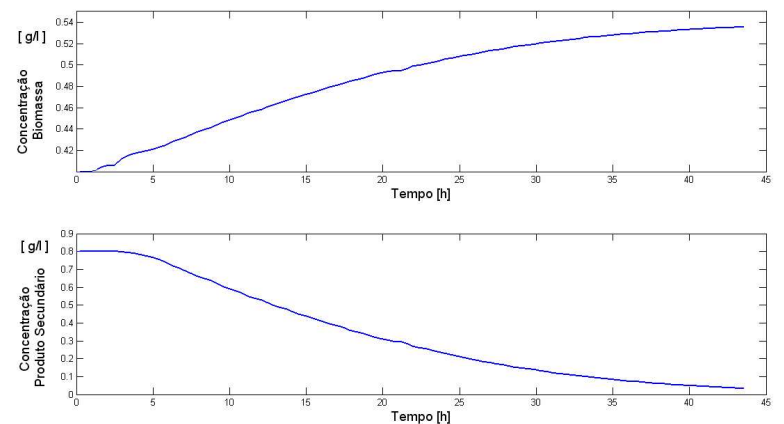

Figura 5: E. Coli - concentrações de biomassa e produto secundário em malha aberta.
Tabela 3: Parâmetros do modelo para S. Cerevisiae.

\begin{tabular}{|c|c|c|}
\hline Coeficientes & Valores & Unidades \\
\hline$k_{1}$ & 0,49 & $g$ de $X / g$ de $S$ \\
\hline$k_{2}$ & 0,05 & $g$ de $X / g$ de $S$ \\
\hline$k_{3}$ & 0,72 & $g$ de $X / g$ de $P$ \\
\hline$k_{4}$ & -1 & - \\
\hline$k_{5}$ & -1 & - \\
\hline$k_{6}$ & 0 & - \\
\hline$k_{7}$ & 0 & - \\
\hline$k_{8}$ & 0,48 & $g \operatorname{de} X / g$ de $S$ \\
\hline$k_{9}$ & -1 & - \\
\hline$k_{10}$ & $-0,3968$ & $g \operatorname{de} X / g$ de $S$ \\
\hline$k_{11}$ & 0 & $g$ de $X / g$ de $S$ \\
\hline$k_{12}$ & $-1,104$ & $g$ de $X / g$ de $P$ \\
\hline$k_{13}$ & 0,5897 & $g$ de $X / g$ de $S$ \\
\hline$k_{14}$ & 0,4621 & $g$ de $X / g$ de $S$ \\
\hline$k_{15}$ & 0,6249 & $g \operatorname{de} X / g$ de $P$ \\
\hline$\mu_{O}$ & 0,256 & $g \mathrm{de} \mathrm{O}_{2} / g$ de $X / \mathrm{h}$ \\
\hline$\mu_{S}$ & 3,5 & $g$ de $S / g$ de $X / h$ \\
\hline$K_{O}$ & 0,0001 & $\mathrm{~g} d \mathrm{e} \mathrm{O}_{2} / \mathrm{l}$ \\
\hline$K_{S}$ & 0,1 & $g$ de $S / l$ \\
\hline$K_{P}$ & 0,1 & g de $P / 1$ \\
\hline$K_{i, P}$ & 10 & $g$ de $P / 1$ \\
\hline
\end{tabular}

Tabela 4: Estimativas dos valores médio e desvio de $r_{1}$.

\begin{tabular}{|c|c|c|}
\hline Parâmetros & E. Coli & S. Cerevisiae \\
\hline$r_{1_{\max }}$ & 0,3205 & 0,5576 \\
\hline$r_{1_{\min }}$ & 0,3185 & 0,4624 \\
\hline $\bar{r}_{1}$ & 0,3195 & 0,5100 \\
\hline$\hat{r}_{1}$ & 0,0010 & 0,0476 \\
\hline
\end{tabular}

na sensibilidade do sensor do produto secundário. Em todos os resultados apresentados nesta seção, supõe-se por simplicidade que o valor de referência do produto secundário seja zero (i.e., $x_{3}^{*}=0$ ).

Primeiramente, considera-se a lei de controle proposta em (26) como ilustrado na Figura 7. Com base nos valores apresentados na Tabela 4, determinou-se numericamente o valor do parâmetro $\lambda$ considerando o problema de otimização definido em (35). A solução foi obtida utilizando o parser YALMIP e o solver SDPT3 levando aos seguintes resultados: $(i) \lambda=0,0026$ e $\alpha=1$ para a bactéria $E$. Coli; e $(i i)$ $\lambda=0,1511$ e $\alpha=1$ para a levedura S. Cerevisiae.

As Figuras 8 e 9 apresentam respectivamente as simulações para as culturas da bactéria e da levedura considerando o sistema em malha fechada com a lei de controle definida em (26). Nota-se que os tempos de produção dos dois processos são diferentes sendo 46,10 horas para a E. Coli, e 33,17 horas para a $S$. Cerevisiae. Este comportamento já era esperado, 


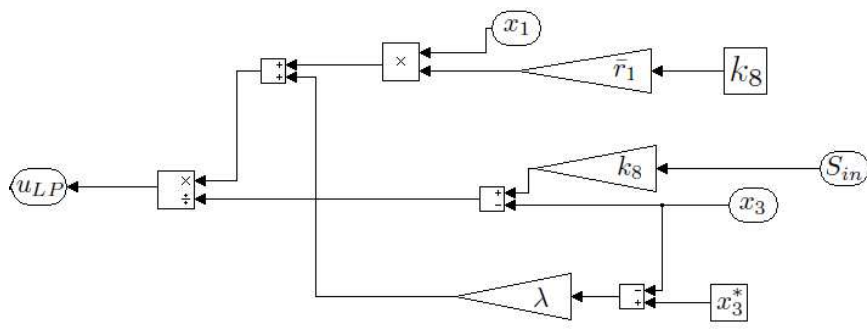

Figura 7: Diagrama em malha fechada no Simulink para a lei de controle $u_{L P}$.
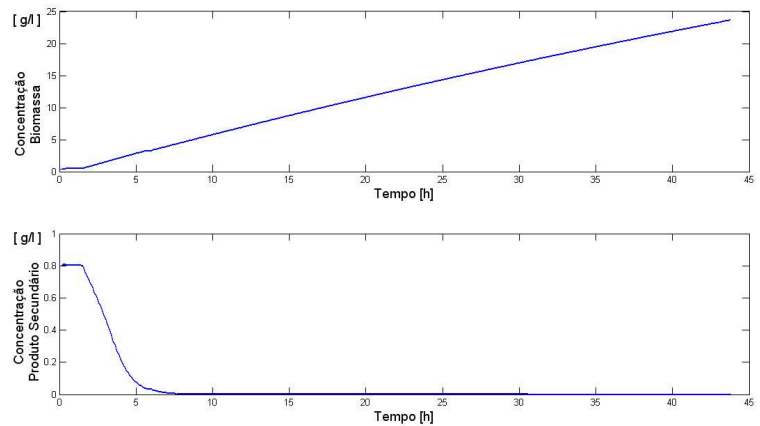

Figura 8: E. Coli - concentrações de biomassa e produto secundário para a lei de controle $u_{L P}$.

pois os parâmetros do modelo dinâmico das duas culturas são diferentes, bem como o volume inicial dos dois bioreatores. As simulações são suspensas quando se chega ao limite do volume de cada bioreator ( 5 litros para a bactéria e 20 litros para a levedura). Os valores da concentração final de biomassa são $24,02 \mathrm{~g} / \mathrm{l}$ para a $E$. Coli e $113,5 \mathrm{~g} / \mathrm{l}$ para a $S$. Cerevisiae. Em ambas as simulações, o objetivo de controle é atingido, pois o valor do erro de seguimento de referência, definido como $x_{3}^{*}-x_{3}$, tende assintoticamente a zero.

As próximas simulações são utilizadas para avaliar o desempenho da lei de controle $u_{L P V}$ (dependente de parâmetros) como definida em (37), sendo a implementação desta lei de controle na plataforma Simulink ilustrada na Figura 10.

Os valores dos parâmetros de sintonia do mecanismo de estimação da taxa $r_{1}$ foram obtidos através de diversas simulações, chegando aos valores $\phi_{1}=-0,00001$ e $\phi_{2}=0,001$ para a bactéria $E$. Coli e $\phi_{1}=-0,1, \phi_{2}=0,09$ para a levedura $S$. Cerevisiae. Para ilustrar o comportamento do mecanismo de estimação de $r_{1}$, apresentam-se nas Figuras 11 e 12 a resposta no tempo da taxa de crescimento real $r_{1}$ e de sua estimativa $\tilde{r}_{1}$ para, respectivamente, a bactéria $E$. Coli e a levedura $S$. Cerevisiae e com isso sendo possível concluir que as duas estimativas estão bem próximas aos valores reais.
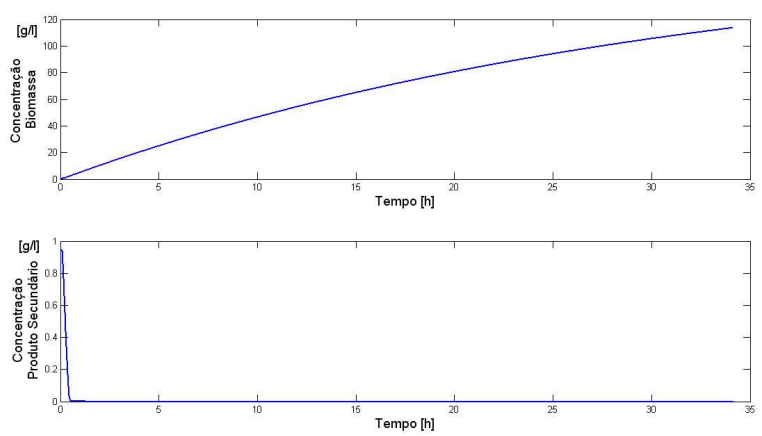

Figura 9: S.Cerevisiae - concentrações de biomassa e produto secundário para a lei de controle $u_{L P}$.

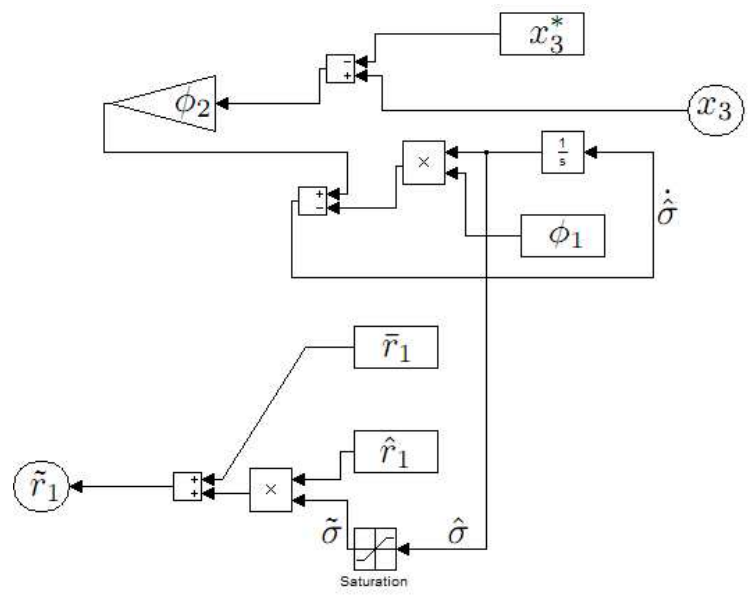

Figura 10: Digrama de simulação para a lei de controle $u_{L P V}$.

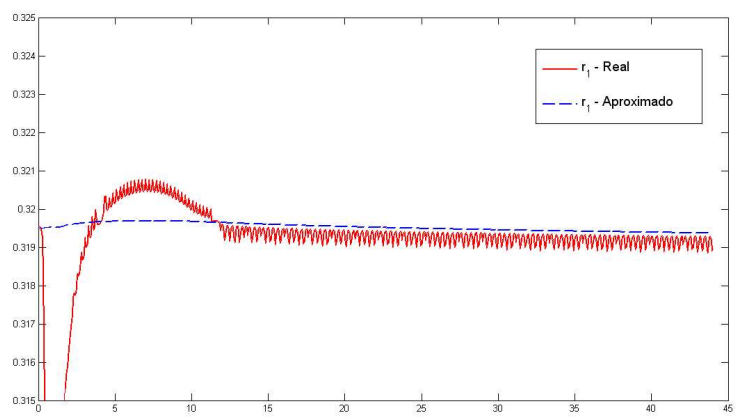

Figura 11: E. Coli- $r_{1}$ e sua estimativa $\tilde{r}_{1}$. 


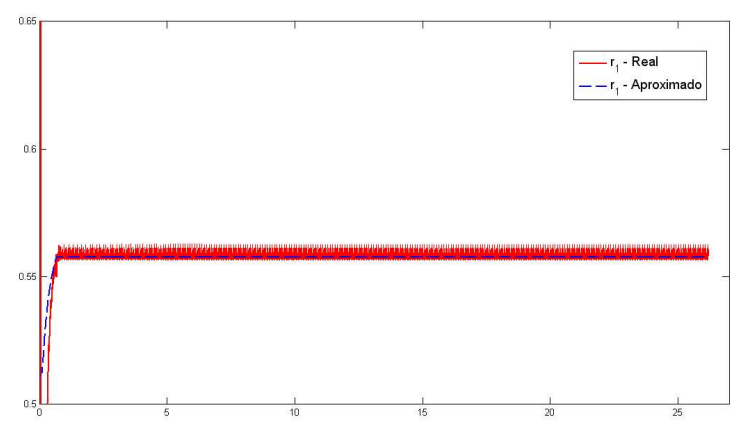

Figura 12: S. Cerevisiae $-r_{1}$ e sua estimativa $\tilde{r}_{1}$.
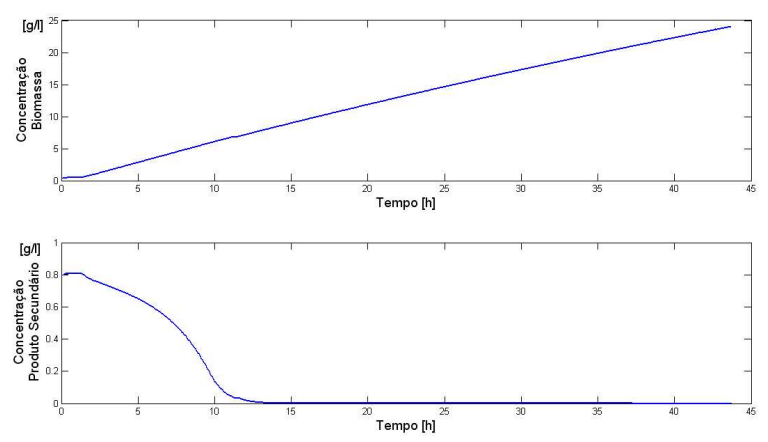

Figura 13: E. Coli - biomassa e produto secundário para lei de controle $u_{L P V}$.

As simulações, considerando a lei de controle $u_{L P V}$, são apresentadas nas Figuras 13, para a bactéria E. Coli, e 14 para a levedura $S$. Cerevisiae. Nota-se claramente que existe uma melhor resposta dinâmica, pois para a $E$. Coli, obtevese o valor máximo da biomassa $24,05 \mathrm{~g} / \mathrm{l}$ em 43,78 horas e para a levedura $S$. Cerevisiae a concentração máxima de biomassa é de 113,5 g/l em 26,19 horas. Em outras palavras, o bioreator utilizando a lei de controle $u_{L P}$ levaria três horas a mais para atingir o mesmo volume de biomassa para a E. Coli e oito horas a mais para a $S$. Cerevisiae quando comparado com a lei dependente de parâmetros $u_{L P V}$. Ressalta-se que os resultados obtidos com a lei de controle $u_{L P V}$ possuem um desempenho compatível com os resultados apresentados nas referências (Chen et al., 1995; Coutinho et al., 2009; Renard e Vande Wouwer, 2008), considerando uma lei de controle menos complexa e com um número restrito de medições online, demonstrando a eficiência da metodologia proposta no projeto de controladores para a maximização de biomassa em bioreatores em modo de operação descontínua com alimentação.
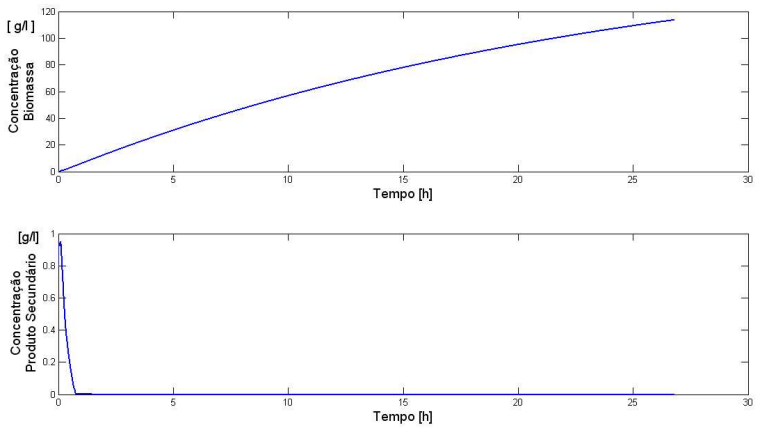

Figura 14: S. Cerevisiae - biomassa e produto secundário para lei de controle $u_{L P V}$.

\section{CONCLUSÕES}

Este artigo apresentou uma abordagem para controle de bioreatores em modo de operação descontínuo com alimentação. A técnica proposta utiliza o conceito de realimentação linearizante parcial, onde a dinâmica livre é projetada de maneira a assegurar uma certa robustez a lei de controle além de atingir um certo desempenho frente a perturbações externas e no modelo. Visando melhorar a resposta dinâmica do controlador com um reduzido número de sensores, apresentou-se um mecanismo simples de estimação de parâmetros para a implementação de uma lei de controle dependente de parâmetros (gain-scheduling). As leis de controle foram aplicadas a dois bioreatores distintos com o objetivo de maximizar a produção de biomassa, considerando as culturas de microorganismos mais utilizados na moderna indústria de biotecnologia. Diversas simulações foram apresentadas para analisar o desempenho do sistema em malha fechada para culturas da bacteria $E$. Coli e da levedura $S$. Cerevisiae, verificando-se o bom comportamento das duas leis de controle propostas. Como sugestão de trabalho futuro, um melhor estudo da técnica de estimação da taxa de reação $r_{1}$ é necessário no que se refere à sintonia dos parâmetros do estimador proposto.

\section{AGRADECIMENTOS}

O primeiro autor deste trabalho agradece à Coordenação de Aperfeiçoamento de Pessoal de Nível Superior - (CAPES) pelo suporte financeiro e a Pontifícia Universidade Católica do Rio Grande do Sul - (PUCRS) pela estrutura fornecida para o desenvolvimento deste projeto. O segundo autor foi parcialmente financiado pelo $\mathrm{CNPq}$, projeto $\mathrm{n}$. 301461/2008-2/PQ, e 200018/2008-6. 


\section{REFERÊNCIAS}

Apkarian, P., Biannic, J.-M. e Gahinet, P. (1995). Gainscheduled $H_{\infty}$ control of a missile via linear matrix Inequalities, Journal of Guidance, Control, and Dynamics 18(3): 532-538.

Bastin, G. e Dochain, D. (1990). On-line estimation and adaptive control of bioreactors, Elsevier.

Bastin, G. e Van Impe, J. F. (1995). Nonlinear and adaptive control in biotechnology: a tutorial, European Jounal of Control 1: 37 - 53.

Boyd, S., El Ghaoui, L., Feron, E. e Balakrishnan, V. (1994). Linear matrix inequalities in system and control theory, SIAM - Society for Industrial and Applied Mathematics.

Chen, L., Bastin, G. e Breusegem, V. (1995). A case study of adaptive nonlinear regulation of fed-batch biologica reactors, Automatica 31: 55-65.

Coutinho, D., Dewasme, L. e Vande Wouwer, A. (2009). Robust control of yeast fed-batch cultures for productivity enhancement, Preprints of the International Symposium on Advanced Control of Chemical Processes (ADCHEM'09), Istambul, Turquia.

Coutinho, D. F. (2003). Análise de estabilidade e desempenho, e sintese de controle para sistemas não lineares incertos, PhD thesis, UFSC - Universidade Federal de Santa Catarina.

Coutinho, D. F., P. D. e Trofino, A. (2004). On the estimation of robust stability regions for nonlinear systems with saturation, Revista Controle e Automação 15(3): 269278.

Coutinho, D. F., Trofino, A. e Fu, M. (2002). Guaranteed cost control of uncertain nonlinear system via polynomial lyapunov functions, IEEE Transactions on Automatic control 47(9): 1575-1580.

Coutinho, D. e Trofino, A. (2002). Análise de sistemas nãolineares incertos: uma abordagem LMI, Revista Controle \& Automação 13(2): 94-104.

Coutinho, D. e Vande Wouwer, A. (2010). A robust $\mathrm{H}_{\infty}$ quasi-LPV approach for designing nonlinear observers, in M. Diehl, F. Glineur, E. Jarlebring e W. Michiels (eds), Recent Advances in Optimization and its Applications in Engineering, Springer Berlin Heidelberg, pp. 21-30.

da Rocha, I. (2003). Model-based strategies for computeraided operation of recombinant $E$. Coli fermentation, $\mathrm{PhD}$ thesis, Universidade do Minho.
Dewasme, L. e Vande Wouwer, A. (2008). Adaptive extremum-seeking control applied to productivity optimization in yeast fed-batch cultures, Proceedings of the 17th IFAC World Congress, Seoul, Coréia do Sul, pp. 9713-9718.

Dewasme, L., Vande Wouwer, A. e Coutinho, D. (2010). Lineazing control of yeast and bacteria fed-batch cultures, 7th International Conference on Infomatics in Control, Automation and Robotics (ICINCO'10), Funchal, Madeira, Portugal.

Dewasme, L., Vande Wouwer, A., Srinivasan, B. e Perrier, M. (2009). Adaptive extremum-seeking control of fed-batch cultures of micro-organisms exhibiting overflow metabolism, Preprints of the International Symposium on Advanced Control of Chemical Processes (ADCHEM'09), Istambul, Turquia.

El Ghaoui, L. e Niculescu, L. I. (2000). Advances in linear matrix inequality methods in control, SIAM - Society for Industrial and Applied Mathematics.

El Ghaoui, L. e Scorletti, G. (1996). Control of rational system using linear-fractional representation and linear matrix inequalities, Automatica 32: 1273-1284.

El Moubaraki, J., Bastin, G. e Levine, J. (1993). Nonlinear control of biotechnological processes with growth-production decoupling, Mathematical Biosciences 116: 21-44.

Freeman, R. e Kokotović, P. (1997). Robust nonlinear control design, Birkhäuser.

Hafidi, G., Tebbani, D., Dumur, D. e Vande Wouwer, A. (2008). Robustness analysis of nonlinear predictive control of fed-batch E. Coli cultures, 16th Mediterranean Conference on Control and Automation.

Hafidi, G., Tebbani, S., Dumur, D. e Vande Wouwer, A. (2007). Towards nonlinear model predictive control of fed-batch cultures of E. Coli, Proc. IEEE International Conference on Control Applications CCA 2007, pp. 194-199.

Henson, M. A. (2006). Biochemical reactor modeling and control, IEEE Control System Magazine 26(4): 54-62.

Henson, M. e Seborg, D. (1997). Feedback linearizing control, in M. Henson e D. Seborg (eds), Nonlinear process control, Prentice-Hall, Upper Saddle River, NJ, pp. 149 -232 .

Huang, Y. e Jadbabaie, A. (1999). Nonlinear $H_{1}$ control: an enhanced quasi-LPV approach, In Proceedings of the IFAC World Congress, pp. 85-90. 
Jenzch, M., Simutis, R. e Luebbert, A. (2006). Generic model control of the specific growth rate in recombinant escherichia coli cultivation, Journal of Biotechnology 122: 483-493.

Lages, W. F. (2009). Controle de robôs móveis, Technical report, Apostila - ELE00070 Tópicos Especiais em Controle e Automação I, Programa de Pós-Graduação em Engenharia Elétrica, UFRGS.

Nagabhushan, T. e Narula, S. (2007). Genetic engineering, in W.-V. V. G. . C. KGaA (ed.), Ullmann's biotechnology and biochemical engineering, Vol. 2, Wiley-VCH Verlag GmbH \& Co. KGaA.

Papachristodoulou, A. e Prajna, S. (2005). A tutorial on sum of squares techniques for systems analysis, Proc. Am. Contr. Conf., Portland, OR.

Renard, F. e Vande Wouwer, A. (2008). Robust adaptive control of yeast fed-batch cultures, Computers and Chimical Engineering 32: 1238 - 1248.

Roeva, O. e Tzonkov, S. (2005). Optimal feed rate control of escherichia coli fed-batch fermentation, Bioautomation 2: 30-36.

Rohr, E., Pereira, L. e Coutinho, D. (2009). Robustness analysis of nonlinear systems subject to state feedback linearization, Revista Controle \& Automação 20(4): $482-489$.

Schügerl, K. e Bellgardt, K. (2000). Bioreaction engineering - modeling and control, Springer-Verlang Berlin Heidelberg.

Slotine, J.-J. e Li, W. (1991). Applied nonlinear control, Prentice Hall.

Smets, I. Y., Claes, J. E., November, E. J., Bastin, G. P. e Impe, J. F. V. (2004). Optimal adaptive control of (bio)chemical reactor: past, present and future, Jounal of Process Control 14: 795 - 805.

Sonnleitner, B. e Käppeli, O. (1986). Growth of saccharomyces cerevisiae is controlled by its limited respiratori capacity: formulation and verification of a hypothesis, $B i$ otechnology and Bioengeneering 28(6): 927-937.

Stephanopoulos, G., Aristidou, A. A. e Nielsen, J. (1998). Metabolic engineering: principles and methodologies, Academic Press.

Tortora, G. J., B.R., F. e Case, C. L. (2008). Microbiologia, Artmed.

Trofino, A. (2000). Notas de aula de controle robusto, Laboratório de controle e micro-informática - Universidade Federal de Santa Catarina.
Van Impe, J. F. e Bastin, G. (1995). Optimal adaptive control of fed-batch fermentation processes, Control Eng. Practice 3: 939 - 954. 